Pacific Journal of Mathematics

THE CLASS OF RECURSIVELY ENUMERABLE SUBSETS OF A
RECURSIVELY ENUMERABLE SET 


\title{
THE CLASS OF RECURSIVELY ENUMERABLE SUBSETS OF A RECURSIVELY ENUMERABLE SET
}

\author{
LOUISE HAY
}

\begin{abstract}
For any set $\alpha$, let $\theta A^{\alpha}$ denote the index set of the class of all recursively enumerable (r.e.) subsets of $\alpha$ (i.e., if $\left\{W_{x}\right\}_{x \geqq 0}$ is a standard enumeration of all r.e. sets, $\theta A^{\alpha}=$ $\left\{x \mid W_{x} \subset \alpha\right\}$.) The purpose of this paper is to examine the possible Turing degrees of the sets $\theta A^{\alpha}$ when $\alpha$ is r.e. It is proved that if $b$ is any nonrecursive r.e. degree, the Turing degrees of sets $\theta A^{\alpha}$ for $\alpha$ r.e., $\alpha \in b$, are exactly the degrees $c>0^{\prime}$ such that $c$ is r.e. in $b$.
\end{abstract}

Index sets of form $\theta A^{\alpha}$ appear to have useful properties in the study of the partial ordering of all index sets under one-to-one reducibility. For instance, in the case where $\alpha$ is a nonrecursive incomplete r.e. set, the index set $\overline{\theta A^{\alpha}}$ was used in [1] to provide an example of an index set which is neither r.e. nor productive. In [2] it is shown that if the Turing degree of $\alpha$ is not $\geqq 0^{\prime}$, then the set $\theta A^{\alpha}$ is at the bottom of $c$ discrete $\omega$-sequences of index sets (i.e., linearly ordered chains of index sets such that no index sets are intermediate between the elements of the chain.) In particular, such a set $\theta A^{\alpha}$ has at least two nonisomorphic immediate successors in the partial ordering of index sets.

It is natural to ask: What relation, if any, exists between the Turing degree of $\alpha$ and that of $\theta A^{\alpha}$ ? In the case where $\alpha$ is co-r.e., it is easy to see that neither degree determines the other, since $\overline{\theta A^{\alpha}}$ is r.e. and hence has degree 0 or $0^{\prime}$ (by Rice's theorem [5]), independently of the degree of $\alpha$; while both 0 and $0^{\prime}$ contain sets $\theta A^{\alpha}$ for $\alpha \in \mathbf{0}$. In this paper it is shown that when $\alpha$ is r.e., the situation is similar, though more complicated. It was shown in [3, Theorem 1] that if $\beta$ is a complete r.e. set, then $\theta A^{\beta}$ is a complete $\Pi_{2}^{0}$ set. On the other hand, C. G. Jockush, Jr. has constructed an example (unpublished) of an effectively simple set $\gamma$ such that $\theta A^{r}$ has degree $0^{\prime}$. Since $\beta$ and $\gamma$ both have degree $0^{\prime}$ [4], this shows that when $\alpha$ is r.e., the degree of $\alpha$ need not determine that of $\theta A^{\alpha}$. The main result of this paper shows that these examples are extremal cases of the fact that when $\alpha$ is r.e., the degree of $\theta A^{\alpha}$ can take on all possible values within certain obvious restrictions. More precisely, we prove the following:

Theorem. Let $\boldsymbol{b}$ be a nonrecursive r.e. degree. Let 
$\mathscr{B}_{1}=\left\{\boldsymbol{c} \mid(\exists \alpha)\left(\alpha \in \boldsymbol{b}\right.\right.$ and $\left.\theta A^{\alpha} \in \boldsymbol{c}\right\}$,

$\mathscr{B}_{2}=\left\{\boldsymbol{c} \mid(\exists \alpha)\left(\alpha\right.\right.$ is r.e. and $\alpha \in \boldsymbol{b}$ and $\left.\theta A^{\alpha} \in \boldsymbol{c}\right\}$,

$\mathscr{B}_{3}=\left\{\boldsymbol{c} \mid \boldsymbol{c} \geqq \mathbf{0}^{\prime}\right.$ and $\boldsymbol{c}$ is r.e. in $\left.\boldsymbol{b}\right\}$.

Then $\mathscr{B}_{1}=\mathscr{B}_{2}=\mathscr{B}_{3}$.

Proof. Clearly $\mathscr{B}_{2} \subset \mathscr{B}_{1}$. It is thus sufficient to prove that $\mathscr{B} \mathscr{B}_{1} \subset \mathscr{B}_{3} \subset \mathscr{B}_{2}$.

$\mathscr{B}_{1} \subset \mathscr{B}_{3}:$ Assume $\alpha \in \boldsymbol{b}$ and $\theta A^{\alpha} \in \boldsymbol{c}$. Since $\overline{\theta A^{\alpha}}=\left\{x \mid W_{x} \cap \bar{\alpha} \neq \varnothing\right\}, \overline{\theta A^{\alpha}}$ is r.e. in $\alpha$ so $c$ is r.e. in $b$. Since $\boldsymbol{b}>\mathbf{0}, \alpha \neq \varnothing$ or $N$, so $\theta A^{\alpha}$ is a nontrivial index set which, by the proof of Rice's theorem [5, Theorem 14-XIV] implies $K \leqq_{T} \theta A^{\alpha}$ (where $K$ denotes the complete r.e. set). So $c \geqq 0^{\prime}$. Since $c$ was arbitrary, this shows $\mathscr{B}_{1} \subset \mathscr{B}_{3}$.

The remainder of this paper is devoted to proving that $\mathscr{\mathscr { B }}_{3} \subset \mathscr{B}_{2}$. We assume that $\boldsymbol{c} \geqq \boldsymbol{0}^{\prime}, \boldsymbol{c}$ r.e. in $\boldsymbol{b}$, and describe the construction of an r.e. set $\alpha$ such that $\alpha \in \boldsymbol{b}$ and $\theta A^{\alpha} \in \boldsymbol{c}$.

2. Preliminaries. The notation is that of [5]. Given $\beta$ r.e., nonrecursive, and $\gamma$ r.e. in $\beta, 0^{\prime} \leqq{ }_{T} \gamma$, we require an r.e. set $\alpha$ such that $\alpha \equiv{ }_{T} \beta$ and $\theta A^{\alpha} \equiv_{T} \gamma$. We attempt to achieve this as follows:

(a) to get $\beta \leqq{ }_{T} \alpha$, we "code" $\beta$ into $\alpha$;

(b) to get $\alpha \leqq_{T} \beta$, we arrange that an odd integer $y$ is put into $\alpha$ only when some $x \leqq y$ has just appeared in $\beta$. (The idea here is similar to that used in the proof of Theorem 2 of [7].);

(c) to get $\gamma \leqq_{T} \theta A^{\alpha}$, we define a sequence $\left\{S_{e}\right\}_{e \geqq 0}$ of r.e. sets such that the index of $S_{e}$ is recursive in $\theta A^{\alpha}$ and $e \in \gamma \leftrightarrow S_{e} \cap \bar{\alpha} \neq \varnothing$;

(d) to get $\theta A^{\alpha} \leqq{ }_{T} \gamma$, we try to "preserve" nonempty intersections $W_{e} \cap \bar{\alpha}$ whenever they occur during the construction.

These requirements evidently conflict, and priorities must be assigned, in the manner of [6].

The fact that $\gamma$ is r.e. in $\beta$ will be used in the following way: Let $\left\{D_{i}\right\}_{i \geqq 0}$ be the canonical indexing of finite sets; $\langle x, y\rangle$ is a standard recursive pairing function with recursive inverses $\pi_{1}, \pi_{2}$, and $\langle x, y, u, v\rangle=\langle\langle\langle x, y\rangle, u\rangle, v\rangle$.

Lemma 1. If $\gamma$ is r.e. in a set $\beta$, then there is a recursive function $f$ such that for each $x, x \in \gamma \leftrightarrow(\exists z)\left(z \in W_{f(x)}\right.$ and $D_{\pi_{1}(z)} \subset \beta$ and $\left.D_{\pi_{2}(z)} \subset \bar{\beta}\right)$.

Proof. Let $\gamma=W_{e}^{\beta}$. Then in the notation of Chapter 9 of [5], $x \in \gamma \leftrightarrow x \in W_{e}^{\beta} \leftrightarrow \varphi_{e}^{\beta}(x)$ is defined $\leftrightarrow(\exists y)(\exists u)(\exists v)\left(\langle x, y, u, v\rangle \in W_{\rho(e)}\right.$ and $D_{u} \subset \beta$ and $\left.D_{V} \subset \bar{\beta}\right)$ where $\rho(e)$ is a recursive function of $e$. Let

$$
V=\left\{\langle u, v\rangle \mid(\exists y)\left(\langle x, y, u, v\rangle \in W_{\rho(e)}\right)\right\} \text {. }
$$


Then $V$ is an r.e. set, whose index can be uniformly computed from $x$; so there is a recursive function $f$ such that $V=W_{f(x)}$, and

$$
\begin{aligned}
x \in \gamma & \longleftrightarrow(\exists u)(\exists v)\left(\langle u, v\rangle \in V \text { and } D_{u} \subset \beta \text { and } D_{v} \subset \bar{\beta}\right) \\
& \longleftrightarrow(\exists z)\left(z \in W_{f(x)} \text { and } D_{\pi_{1}(z)} \subset \beta \text { and } D_{\pi_{2}(z)} \subset \bar{\beta}\right) .
\end{aligned}
$$

Definition 2. Let $g$ be a recursive function such that $\left\{D_{g(i)}\right\}_{i \geqq 0}$ is a recursive partitioning of the positive even integers into disjoint finite sets such that $\left|D_{g(i)}\right|=i+1$ for each $i$ (e.g., let $D_{g(i)}=$ $\left.\left\{i^{2}+i+2 k \mid 0<k \leqq i+1\right\}\right)$. Let $e_{x}=e(x)$ be a recursive function such that $e_{x}=$ the unique $i$ for which $2 x \in D_{g(i)}$.

3. Construction. $\alpha$ will be constructed in stages, $\alpha=\mathrm{U}_{s} \alpha_{s}$ where $\alpha_{s}$ is the finite set of integers which has been put into $\alpha$ by the end of stage $s$. If $W$ is r.e., $W^{s}$ will denote the result of performing $s$ steps in some fixed enumeration of $W$; in particular $W^{0}=\varnothing$.

We define $\alpha_{s}$ and auxiliary recursive functions $y_{e}^{s}=y_{e}(s)$ and $z_{e}^{s}=z_{e}(s)$ and a partial recursive function $h(y)$ by simultaneous recursion. If $y_{e}^{s}>0, y_{e}^{s}$ serves to witness that $e \in \gamma$, while $z_{e}^{s}$ witnesses that $W_{e} \cap \bar{\alpha} \neq \varnothing$.

Stage 0.

$$
\alpha_{0}=\{0\}, y_{e}^{0}=z_{e}^{0}=0 \text {. }
$$

Let $C_{s}=\left\{z \mid z>0\right.$ and $\left.(\exists e)(\exists t)_{t<s}\left(z=y_{e}^{s} \vee z=z_{e}^{t}\right)\right\}$; so $C_{1}=\varnothing$. Assume inductively that $C_{s}$ is finite and that $y_{e}^{s-1}>0$ implies $y_{e}^{s-1}$ is odd and $h\left(y_{e}^{s-1}\right)$ is defined, for all $e$.

Stage $s>0, s \equiv 1(\bmod 3)$.

Let

$$
\begin{aligned}
& E_{s}=\left\{y \mid(\exists x)\left(y=2 x \text { and } e_{x} \leqq s \text { and } e_{x} \in \beta^{s} \text { and }(\forall i)_{i<e_{x}}\left(y \neq z_{i}^{s-1}\right)\right\}\right. \\
& O_{s}=\{y \mid \exists e)_{e \leqq s}\left(y=y_{e}^{s-1} \text { and } D_{\pi_{2}(h(y))} \cap \beta^{s} \neq \varnothing\right\} .
\end{aligned}
$$

Let $\alpha_{s}=\alpha_{s-1} \cup E_{s} \cup O_{s}$. If $z_{e}^{s-1} \in E_{s}$, let $z_{e}^{s}=0$. Otherwise, let $z_{e}^{s}=z_{e}^{s-1}$. If $y_{e}^{s-1} \in O_{s}$, let $y_{e}^{s}=0$. Otherwise, let $y_{e}^{s}=y_{e}^{s-1}$.

Stage $s>0, s \equiv 2(\bmod 3)$.

Let $\alpha_{s}=\alpha_{s-1}$. For each $e \leqq s$ (if any) such that

(a) $z_{e}^{s-1}=0$ and

(b) $(\exists z)\left(z \in W_{e}^{s} \cap \bar{\alpha}_{s}\right.$ and $(\forall i)_{i \leqq e}\left(z \neq y_{i}^{s-1}\right)$ and $\left.(\forall x)_{x<z}\left(z=2 x \rightarrow e_{x}>e\right)\right)$,

let $z_{e}^{s}=$ the least $\operatorname{such} z$. For all other $e$, let $z_{e}^{s}=z_{e}^{s-1}$. If $y_{j}^{s-1}=z_{e}^{s}$ 
for some $e<j$, let $y_{j}^{s}=0$. Otherwise let $y_{j}^{s}=y_{j}^{s-1}$.

Stage $s>0, s \equiv 0(\bmod 3)$.

Let $\alpha_{s}=\alpha_{s-1}, z_{e}^{s}=z_{e}^{s-1}$ for all $e$. Let $F_{s}=\left\{e \mid e \leqq s\right.$ and $y_{e}^{s-1}=0$ and $(\exists x)\left(x \in W_{f(e)}^{s}\right.$ and $D_{\pi_{1}(x)} \subset \beta^{s}$ and $\left.\left.D_{\pi_{2}(x)} \subset \overline{\beta^{s}}\right)\right\}$. If $e \notin F_{s}$, let $y_{e}^{s}=y_{e}^{s-1}$. If $F_{s} \neq \varnothing$, let $F_{s}=\left\{k_{0}, k_{1}, \cdots, k_{n}\right\}, n \geqq 0, k_{i}<k_{j}$ for $i<j$. Define $y_{k_{i}}^{s}$ inductively as follows: assume $y_{k_{j}}^{s}$ has been defined for all $j<i$. Let $x_{i}=$ least $x$ such that $x \in W_{f\left(k_{i}\right)}^{s}$ and $D_{\pi_{1}(x)} \subset \beta^{s}$ and $D_{\pi_{2}(x)} \subset \overline{\beta^{s}}, y_{k_{i}}^{s}=$ least odd $y \in \bar{\alpha}_{s}$ such that

$$
y>\max \left(D_{\pi_{1}\left(x_{i}\right)} \cup D_{\pi_{2}\left(x_{i}\right)} \cup C_{s} \cup\left\{y_{k_{j}}^{s} \mid j<i\right\}\right) .
$$

Define $h\left(y_{k_{i}}^{s}\right)=x_{i}$ for each $i \leqq n$.

It is easily verified for all three types of stages that $C_{s}$ is always finite, since new nonzero values are assigned to $z_{e}^{s}$ and $y_{e}^{s}$ for at most $s+1$ values of $e$. For the second inductive assumption, it suffices to note that new nonzero values of $y_{e}^{s}$ are defined only if $s \equiv 0(\bmod 3)$, and that each new value $y_{e}^{s}>0$ is odd and $h\left(y_{e}^{s}\right)$ is defined. That $h(y)$ is well defined will be proved below.

It is clear that $\alpha=\bigcup_{s} E_{s} \cup \bigcup_{s} O_{s} \cup\{0\}$ is r.e. We note for later use that $O_{s}$ consists of odd numbers and $E_{s}$ of even numbers, so that

$$
\begin{gathered}
0<y=2 x \in \alpha \longleftrightarrow(\exists s)\left(s \equiv 1(\bmod 3) \text { and } y \in E_{s}\right), \\
y \text { odd }, y \in \alpha \longleftrightarrow(\exists s)\left(s \equiv 1(\bmod 3) \text { and } y \in O_{s}\right) .
\end{gathered}
$$

\section{Proof of Theorem.}

Lemma 3. For all $e$ and $s$,

(a) If $y_{e}^{s-1} \neq y_{e}^{s}$ then either (i) $s \not \equiv 0(\bmod 3), y_{e}^{s-1}>0$ and $y_{e}^{s}=0$, or (ii) $s \equiv 0(\bmod 3), y_{e}^{s-1}=0$ and $y_{e}^{s}>0$.

(b) If $y=y_{e}^{s-1}>0$ and $y_{e}^{s} \neq y$, then either $(\exists i)_{i<e}\left(y=z_{i}^{s}\right)$ or $s \equiv 1$ $(\bmod 3)$ and $y \in O_{s}$.

(c) If $y_{e}^{s}>0$, then either (i) $(\forall t)\left(t>s \rightarrow y_{e}^{t}=y_{e}^{s}\right)$ or (ii) if $t^{\prime}=$ least $t>s$ such that $y_{e}^{t} \neq y_{e}^{s}$ then $y_{e}^{t^{\prime}}=0$ and $\left(\forall s^{\prime}\right)\left(s^{\prime}>t^{\prime} \rightarrow y_{e}^{s^{\prime}}=0\right.$ or $\left.y_{e}^{s^{\prime}}>y_{e}^{s}\right)$.

(d) If $s<t$ and $0<y_{e}^{s}=y_{e}^{t}$, then $\left(\forall t^{\prime}\right)\left(s<t^{\prime}<t \rightarrow y_{e}^{t^{\prime}}=y_{e}^{s}\right)$.

(e) If $\lim _{s} y_{e}^{s}$ exists and $\lim _{s} y_{e}^{s}=y>0$, then $(\forall s)(\forall t)\left(y=y_{e}^{s}\right.$ and $s<t \rightarrow y=y_{e}^{t}$ ).

Proof. (a) is clear from the construction.

(b) Assume $y=y_{e}^{s-1}>0, y_{e}^{s} \neq y$. Then by $(a), s \neq 0(\bmod 3)$. If $s \equiv 1(\bmod 3)$, then $y_{e}^{s} \neq y_{e}^{s-1}$ only if $y_{e}^{s-1} \in O_{s}$. If $s \equiv 2(\bmod 3)$, then $y_{e}^{s} \neq y_{e}^{s-1}$ only if $y=y_{e}^{s-1}=z_{i}^{s}$ for some $i<e$.

(c) Assume $y=y_{e}^{s}>0$. If (i) fails to hold, let $t^{\prime}$ be the least 
$t>s$ such that $y_{e}^{t} \neq y_{e}^{s}$; thus $t^{\prime}>s$ and $0<y=y_{e}^{s}=y_{e}^{t^{\prime}-1} \neq y_{e}^{t^{\prime}}$. Then by (a), $y_{e}^{t^{\prime}}=0$. Suppose (ii) fails to hold; then for some $s^{\prime}>t^{\prime}$, $0<y_{e}^{s^{\prime}} \leqq y$. Let $s^{\prime}$ be least. Then $s^{\prime}-1 \geqq t^{\prime}$ and $y_{e}^{s^{\prime}-1}=0$ or $y_{e}^{s^{\prime}-1}>y>0$. So $y_{e}^{s^{\prime}} \neq y_{e}^{s^{\prime}-1}$, and it follows by (a) that $s \equiv 0(\bmod 3)$ and $y_{e}^{s^{\prime}-1}=0$. But $s^{\prime}>t^{\prime}>s$ implies that $y=y_{e}^{s} \in C_{s^{\prime}}$, while $y_{e}^{s^{\prime}} \neq y_{e}^{s^{\prime}-1}$ implies $y_{e}^{s^{\prime}}>\max C_{s^{\prime}}$. So $y_{e}^{s^{\prime}}>y$, which is a contradiction. So (ii) must hold.

(d) Let $s<t$ and $0<y_{e}^{s}=y_{e}^{t}$. Suppose that $y_{e}^{t^{\prime}} \neq y_{e}^{s}$ for some $t^{\prime}, s<t^{\prime}<t$ and let $t^{\prime}$ be least. Then by (c), $y_{e}^{t^{\prime}}=0$, and $t>t^{\prime}>s$ implies $y_{e}^{t}=0$ or $y_{e}^{t}>y_{e}^{s}$, both contrary to hypothesis. So $y_{e}^{s}=y_{e}^{t}$ implies $y_{e}^{t^{\prime}}=y_{e}^{s}$ for all $t^{\prime}, s<t^{\prime}<t$.

(e) Assume $\lim _{s} y_{e}^{s}=y>0$ and $y=y_{e}^{s}$. Suppose that for some $t>s, y_{e}^{t} \neq y$, and let $t$ be least. Then by (c), $y_{e}^{s^{\prime}} \neq y$ for all $s^{\prime}>t$ which contradicts the assumption that $y=\lim _{s} y_{c}^{s}$.

Lemma 4. For all $e$ and $s$,

(a) $y_{e}^{s}>0$ implies $y_{e}^{s} \in \overline{\alpha_{s}}$.

(b) If $y_{e}^{s}>0$ and $e^{\prime} \neq e$, then $y_{e}^{s} \neq y_{e^{\prime}}^{t}$ for all $t$.

(c) If $y=y_{e}^{s}>0$, then $h(y)$ is well-defined.

Proof. (a) Assume $y=y_{e}^{s}>0$, and let $s^{\prime}$ be the least $t$ such that $y=y_{e}^{t}$. Then $0<s^{\prime} \leqq s$, and $y_{e}^{s^{\prime}-1} \neq y_{e}^{s^{\prime}}=y>0$. So by Lemma $3(a), y_{e}^{s^{\prime}-1}=0$ and $s^{\prime} \equiv 0(\bmod 3)$. By the construction, $y_{e}^{s^{\prime}}>y_{e}^{s^{\prime}-1}$ implies $y_{e}^{s^{\prime}} \in \overline{\alpha_{s^{\prime}}}$. Now assume $y \in \alpha_{s}$, and consider the least $t$ such that $y \in \alpha_{t}$. Clearly $s^{\prime}<t \leqq s, y \in \overline{\alpha_{t-1}}$ and $t \equiv 1(\bmod 3)$. Now $y=$ $y_{\epsilon}^{s}>0$ is odd, so $y \notin E_{t} \cup \alpha_{t-1}$. So $y \in \alpha_{t}$ implies $y \in O_{t}$. By Lemma $3(\mathrm{~d}), y_{e}^{s}=y_{e}^{s^{\prime}}$ and $s^{\prime} \leqq t-1<t \leqq s$ implies $y_{e}^{t-1}=y_{e}^{t}=y$. But by the construction, $y=y_{e}^{t-1} \in O_{t}$ implies $y_{e}^{t}=0 \neq y$, which is a contradiction. So $y \in \overline{\alpha_{s}}$.

(b) Assume $y_{e}^{s}>0$ and $e^{\prime} \neq e$. Clearly if $y_{e^{\prime}}^{t}=0$ then $y_{e^{\prime}}^{t} \neq y_{e}^{s}$; so assume $y_{e^{\prime}}^{t}>0$. Consider the least $s^{\prime}$ such that $y_{e}^{s}=y_{e}^{s^{\prime}}$ and the least $t^{\prime}$ such that $y_{e^{\prime}}^{t}=y_{e^{\prime}}^{t^{\prime}}$. Then $s^{\prime} \equiv t^{\prime} \equiv 0(\bmod 3), e \in F_{s^{\prime}}$ and $e^{\prime} \in F_{t^{\prime}}$. If $s^{\prime}<t^{\prime}$ then $y_{e}^{s^{\prime}} \in C_{t^{\prime}}$, so by the construction,

$$
y_{e^{\prime}}^{t}=y_{e^{\prime}}^{t^{\prime}}>y_{e}^{s^{\prime}}=y_{e}^{s} \text {. }
$$

If $s^{\prime}>t^{\prime}$, then $y_{e^{\prime}}^{s^{\prime}} \in C_{s^{\prime}}$, so $y_{e}^{s}=y_{e}^{s^{\prime}}>y_{e^{\prime}}^{t^{\prime}}=y_{e}^{t}$. If $s^{\prime}=t^{\prime}$, then $e, e^{\prime} \in F_{s^{\prime}}$, $e=k_{i}, e^{\prime}=k_{j}$ for $i \neq j$. If $e<e^{\prime}$ then $i<j$ and $y_{e}^{s}=y_{e}^{s^{\prime}} \in\left\{y_{k_{2}}^{s^{\prime}} \mid i<j\right\}$ while $y_{e^{\prime}}^{t}=y_{e^{\prime}}^{s^{\prime}}>\max \left\{y_{k_{i}}^{s^{\prime}} \mid i<j\right\}$, so $y_{e^{\prime}}^{t}>y_{e}^{s}$. By symmetry, if $e^{\prime}<e$ then $y_{e}^{s}>y_{e^{\prime}}^{t}$. Thus in any case, $e \neq e^{\prime}$ implies $y_{e}^{s} \neq y_{e^{\prime}}^{t}$.

(c) First note that by the construction, $h(y)$ is defined if and only if there exist $e, s$ such that $y_{e}^{s-1}=0$ and $y=y_{e}^{s}>0$. In particular, if $y=y_{e}^{s}>0, h(y)$ is defined since if $s^{\prime}$ is the least $t$ such that $y=y_{e}^{t}$, then $y_{e}^{s^{\prime}-1}=0$. To show $h(y)$ is well-defined, it suffices to 
show that there exists at most one pair $e, s$ such that $y=y_{e}^{s}$ and $y_{e}^{s-1}=0$. Suppose $y=y_{e}^{s}=y_{i}^{t}$ where $y_{e}^{s-1}=y_{i}^{t-1}=0$. Since $y>0$, $y_{e}^{s}=y_{i}^{t}$ implies $i=e$, by part (b) of this lemma. If $s \neq t$, say $s<t$, then $s \leqq t-1<t$. But then by Lemma 3(d), $y_{e}^{t-1}=y_{i}^{t-1}=0 \neq y_{e}^{s}$ implies $y_{e}^{s} \neq y_{e}^{t}=y_{i}^{t}$, contrary to hypothesis. This completes the proof.

Definition 5. Let $\tau(x)=$ the least $t$ such that

$$
(\forall z)_{z \leqq x}\left(z \in \beta \longrightarrow z \in \beta^{t}\right) \text {. }
$$

Then $\tau(x)$ is defined for all $x$, and $\tau(x)$ is evidently recursive in $\beta$.

Lemma 6. If $t \equiv 1(\bmod 3), y=y_{e}^{t-1}>0$ and $D_{\pi_{2} h(y)} \cap \beta^{t} \neq \varnothing$, then $t<\tau(y)+3$.

Proof. Assume the hypothesis. Let $s^{\prime}$ be the least $s$ such that $y=y_{e}^{s}$ and let $x=h(y) ; x$ is well-defined by Lemma $4(\mathrm{c})$. Then $e \leqq s^{\prime}<t, s^{\prime} \equiv 0(\bmod 3)$ and, by the construction, $D_{\pi_{2}(x)} \subset \overline{\beta^{s^{\prime}}}$ and $y=y_{e}^{s^{\prime}}>\max D_{\pi_{2}(x)}$. By hypothesis, $D_{\bar{\pi}_{2}(x)} \cap \beta^{t} \neq \varnothing$; let $z$ be any element of $D_{\pi_{2}(x)} \cap \beta^{t}$. Then $z \in D_{\pi_{2}(x)}$ implies $z<y$, so that $z \in \beta$, $z \notin \beta^{s^{\prime}}$ implies $s^{\prime}<\tau(y)$. Now suppose $t \geqq \tau(y)+3$, and let $s=t-3$. Then $s \geqq \tau(y)>s^{\prime} \geqq e$ and $s \equiv t \equiv 1(\bmod 3)$. Also $s \geqq \tau(y)$ implies $z \in \beta^{s}$, so $D_{\pi_{2}(x)} \cap \beta^{s} \neq \varnothing$. By Lemma 3(d), $0<y=y_{e}^{s^{\prime}}=y_{e}^{t-1}$ and $s^{\prime} \leqq s-1<s<t-1$ implies $y_{e}^{s-1}=y_{e}^{s}=y$. But by the construction, $e \leqq s$ and $y=y_{e}^{s-1}$ and $D_{\pi_{2} h(y)} \cap \beta^{s} \neq \varnothing$ implies $y_{e}^{s-1} \in O_{s}$, so that $y_{e}^{s}=0 \neq y$. Since this is a contradiction, we conclude $t<\tau(y)+3$.

Lemma 7. Assume $y$ is odd. Then $y \in \alpha$ if and only if $(\exists t)_{t<\tau(y)+3}(\exists e)_{e \leqq t}\left(t \equiv 1(\bmod 3)\right.$ and $y=y_{e}^{t-1}$ and $\left.D_{\pi_{2} h(y)} \cap \beta^{t} \neq \varnothing\right)$.

Proof. By the construction, if $y$ is odd then

$$
\begin{aligned}
y \in \alpha & \longleftrightarrow(\exists t)\left(t \equiv 1(\bmod 3) \text { and } y \in O_{t}\right) \\
& \longleftrightarrow(\exists t)(\exists e)_{e \leqq t}\left(t \equiv 1(\bmod 3) \text { and } y=y_{e}^{t-1}\right. \text { and } \\
& \left.D_{\pi_{2} h(y)} \cap \beta^{t} \neq \varnothing\right) .
\end{aligned}
$$

By Lemma 6 , such a $t$ can be bounded by $\tau(y)+3$, which proves the lemma.

LEMMA 8. For all $i$ and $s$,

(a) If $z_{i}^{s-1}>0$ and $z_{i}^{s} \neq z_{i}^{s-1}$, then $s \equiv 1(\bmod 3), z_{i}^{s}=0$ and $z_{i}^{s-1} \in E_{s} \subset \alpha_{s}$. 
(b) If $z=2 x>0, i<e_{x}$ and $z=z_{i}^{\varsigma}$, then $z=z_{i}^{t}$ for all $t \geqq s$.

Proof. (a) Assume $z_{i}^{s-1}>0$ and $z_{i}^{s} \neq z_{i}^{s-1}$. If $s \equiv 0(\bmod 3)$, then $z_{i}^{s}=z_{i}^{s-1}$ for all $i$. If $s \equiv 2(\bmod 3)$ then $z_{i}^{s} \neq z_{i}^{s-1}$ only if $z_{i}^{s-1}=0$. It follows that $s \equiv 1(\bmod 3)$. But then $z_{i}^{s} \neq z_{i}^{s-1}$ only if $z_{i}^{s-1} \in E_{s} \subset \alpha_{s}$, and in that case $z_{i}^{s}=0$.

(b) Assume $z=2 x>0, i<e_{x}$ and $z=z_{i}^{s}$. Suppose $(\exists t)(t>s$ and $z_{i}^{t} \neq z_{i}^{s}$ ), and let $t$ be least. Then $0<z=z_{i}^{t-1} \neq z_{i}^{t}$; so by (a), $t \equiv 1(\bmod 3)$ and $z \in E_{t}$. But this implies that $\left(\exists x^{\prime}\right)\left(z=2 x^{\prime}\right.$ and $\left.(\forall i)_{i<e_{x^{\prime}}}\left(z \neq z_{i}^{t-1}\right)\right)$. Clearly $x^{\prime}=x$, so $(\forall i)_{i<e_{x}}\left(z \neq z_{i}^{t-1}\right)$ which is a contradiction. So $z=z_{i}^{t}$ for all $t \geqq s$.

Definition 9. Define functions $\sigma(y), \sigma^{\prime}(y)$ as follows:

(a) If $y$ is odd, $\sigma^{\prime}(y)=0$.

(b) If $y=2 x$ and $e_{x} \notin \beta, \sigma^{\prime}(y)=0$.

(c) If $y=2 x$ and $e_{x} \in \beta, \sigma^{\prime}(y)=$ least $s$ such that $e_{x} \in \beta^{s}$.

(d) If $\sigma^{\prime}(y)=0$ then $\sigma(y)=0$.

(e) If $\sigma^{\prime}(y)>0$, then $\sigma(y)=$ least $s \geqq \max \left\{e_{x}, \sigma^{\prime}(y)\right\}$ such that $s \equiv 1(\bmod 3)$.

It is clear that $\sigma, \sigma^{\prime}$ are defined for all $y$ and that $\sigma(y)>0$ if and only if $y=2 x$ and $e_{x} \in \beta$. Since $e_{x}$ is a recursive function of $x, \sigma^{\prime}$ and $\sigma$ are recursive in $\beta$.

Lemma 10. Assume $y=2 x>0$. Then $y \in \alpha$ if and only if $e_{x} \in \beta$ and $\sigma(y)>0$ and $(\forall i)_{i<e_{x}}\left(y \neq \boldsymbol{z}_{i}^{\sigma(y)-1}\right)$.

Proof. (๘). Assume $e_{x} \in \beta$ and $\sigma(y)>0$ and $(\forall i)_{i<e_{x}}\left(y \neq z_{i}^{\tau(y)-1}\right)$. Then by Definition $9, e_{x} \leqq \sigma(y)$ and $e_{x} \in \beta^{\sigma^{\prime}(y)} \subset \beta^{\sigma(y)}$. Then by the construction, since $\sigma(y) \equiv 1(\bmod 3), y \in E_{\sigma(y)} \subset \alpha_{\sigma(y)}$. So $y \in \alpha$.

$(\rightarrow)$. Assume $y \in \alpha$. Then since $y>0$ is even, $y \in E_{s}$ for some $s, s \equiv 1(\bmod 3)$; so $e_{x} \in \beta^{s}, e_{x} \leqq s$ and $(\forall i)_{i<e_{x}}\left(y \neq z_{i}^{s-1}\right)$. So in particular $e_{x} \in \beta$, and, by definition of $\sigma(y), 0<\sigma(y) \leqq s$. Suppose that for some $i<e_{x}, y=z_{i}^{\sigma(y)-1}$. Then by Lemma $8(\mathrm{~b}), y=z_{i}^{s-1}$, since

$$
s-1 \geqq \sigma(y)-1 \text {. }
$$

But this is a contradiction, which proves the lemma.

LEMMA 11. $\alpha \leqq{ }_{T} \beta$.

Proof. We show how to decide membership in $\alpha$, recursively in $\beta$. If $y=0$, then $y \in \alpha$, by Stage 0 of the construction. Suppose $y>0$. 
Case 1. $y$ is odd.

Then by Lemma 7, $y \in \alpha$ if and only if $(\exists t)_{t<\tau(y)+3}(\exists e)_{e \leq t}(t \equiv 1$ $(\bmod 3)$ and $y=y_{e}^{t-1}$ and $\left.D_{\pi_{2} h(y)} \cap \beta^{t} \neq \varnothing\right)$. Now for fixed $t, y$ it can be decided recursively whether $t \equiv 1(\bmod 3)$ and whether $y=y_{e}^{t-1}$ for some $e \leqq t$, since $y_{e}^{s}$ is a recursive function of $s$. If $y=y_{e}^{t-1}$, then $h(y)$ is well defined by Lemma $4(\mathrm{c})$, and it can be decided recursively whether $D_{\pi_{2} h(y)} \cap \beta^{t} \neq \varnothing$. So $y \in \alpha \leftrightarrow(\exists t)_{t<\tau(y)+3} R(t, y)$ where $R(t, y)$ is a recursive predicate. Since, as noted in Definition $5, \tau(y)$ is recursive in $\beta$, the question of whether $y \in \alpha$ can be decided, recursively in $\beta$.

Case 2. $y$ is even.

Then by Lemma 10, $y=2 x$ implies that $y \in \alpha$ if and only if $e_{x} \in \beta$ and $\sigma(y)>0$ and $(\forall i)_{i<e_{x}}\left(y \neq z_{i}^{\sigma(y)-1}\right)$. For $y=2 x$, whether $e_{x} \in \beta$ can be decided recursively in $\beta$, since $e_{x}$ is a recursive function of $x$. If $e_{x} \notin \beta$, then $y \notin \alpha$. If $e_{x} \in \beta$, then $\sigma(y)>0$ can be computed recursively in $\beta$, as noted in Definition 9. Since $z_{i}^{s}$ is a recursive function of $s$, the membership of the finite set $D=\left\{z_{i}^{\sigma(y)-1} \mid i<e_{x}\right\}$ can be completely determined, once $\sigma(y)$ is known. Then

$$
y \in \alpha \longleftrightarrow y \notin D \text {. }
$$

Lemma 12. For all $e, e \in \beta \leftrightarrow D_{g(e)} \cap \alpha \neq \varnothing$.

Proof. (↔). Assume $D_{g(e)} \cap \alpha \neq \varnothing$. Let $y \in D_{q(e)} \cap \alpha$. Then $y \in D_{g(e)}$ implies $y$ is even, $y>0$, and, as remarked above, $y \in \alpha$ implies $y \in E_{s}$ for some $s$. So $y=2 x$ for some $x$ such that $e_{x} \in \beta$, i.e., $y=2 x \in D_{g(i)}$ where $i \in \beta$. But by definition of $g, D_{g(e)} \cap D_{g(i)} \neq \varnothing$ implies $i=e$; so $e \in \beta$.

$(\rightarrow)$. Assume $e \in \beta$, and let $t \equiv 1(\bmod 3)$ be so large that $t \geqq e$ and $e \in \beta^{t}$. Now by the definition of $g, D_{g(e)}$ has $e+1$ elements, while $\left\{z_{i}^{t-1} \mid i<e\right\}$ has at most $e$ elements. So $D_{g(e)}-\left\{z_{i}^{t-1} \mid i<e\right\} \neq \varnothing$. Let $y=2 x \in D_{g(e)}-\left\{z_{i}^{t-1} \mid i<e_{x}\right\}$. Then $y>0$, and by definition of $e_{x}, e_{x}=e$. So $e_{x} \leqq t, e_{x} \in \beta^{t}$ and $(\forall i)_{i<e_{x}}\left(y \neq z_{i}^{t-1}\right)$. This implies $y \in E_{t} \subset \alpha_{t}$, and $y \in D_{g(e)} \cap \alpha$.

Lemma 13. $\beta \leqq{ }_{T} \alpha$.

Proof. By Lemma 12, $\beta$ is in fact truth-table reducible to $\alpha$.

Lemma 14. For all $i$, e and $s, z_{e}^{s}>0$ implies $z_{e}^{s} \neq y_{i}^{s}$.

Proof. By induction on $s$. Since $z_{e}^{0}=0$, the lemma holds 
vacuously for $s=0$. Assume that for all $i$ and $e, z_{e}^{s-1}>0$ implies $z_{e}^{s-1} \neq y_{i}^{s-1}$, and assume $y_{e}^{s}>0$.

Case 1. $s \equiv 0(\bmod 3)$.

Then $z_{e}^{s}=z_{e}^{s-1}$, which implies $z_{e}^{s-1}>0$. Then by the induction hypothesis, $z_{e}^{s-1} \neq y_{i}^{s-1}$ for all $i$. If $y_{i}^{s}=y_{i}^{s-1}$, then

$$
z_{e}^{s}=z_{e}^{s-1} \neq y_{i}^{s-1}=y_{i}^{s} .
$$

If $y_{i}^{s} \neq y_{i}^{s-1}$, then by the construction, $z_{e}^{s}=z_{e}^{s-1} \in C_{S}$ while $y_{i}^{s}>\max C_{s}$. So in either case, $z_{e}^{s} \neq y_{i}^{s}$.

Case 2. $s \equiv 1(\bmod 3)$.

By the construction, $z_{e}^{s} \neq z_{e}^{s-1}$ implies $z_{e}^{s}=0$ and $y_{i}^{s} \neq y_{i}^{s-1}$ implies $y_{i}^{s}=0$. So $z_{e}^{s}>0$ implies $z_{e}^{s}=z_{e}^{s-1} \neq y_{i}^{s-1}$, by the induction hypothesis. If $y_{i}^{s-1}=y_{i}^{s}$, clearly $z_{e}^{s} \neq y_{i}^{s}$. If $y^{s} \neq y_{i}^{s-1}$ then $y_{i}^{s}=0$ so $y_{i}^{s} \neq z_{e}^{s}$ since $z_{e}^{\circ}>0$.

Case 3. $s \equiv 2(\bmod 3)$.

For each $i$, either $y_{i}^{s}=y_{i}^{s-1}$ or $y_{i}^{s}=0$. If $y_{i}^{s}=0$ then $y_{i}^{s} \neq z_{e}^{s}$. If $y_{i}^{s}=y_{i}^{s-1}$, then $y_{i}^{s}=z_{e}^{s}$ implies $y_{i}^{s-1}=z_{e}^{s}$. If $z_{e}^{s}=z_{e}^{s-1}$ this contradicts the induction hypothesis, since in that case $z_{e}^{s-1}>0$. So $z_{e}^{s}=y_{i}^{s}=y_{i}^{s-1}$ implies $z_{e}^{s} \neq z_{e}^{s-1}$ which by the construction implies $z_{e}^{s}$ satisfies

$$
(\forall i)_{i \leqq e}\left(z_{e}^{s} \neq y_{i}^{s-1}\right) \text {. }
$$

So $z_{e}^{s}=y_{i}^{s-1}$ implies $i>e$. But by the construction, $z_{e}^{s}=y_{i}^{s-1}$ for $i>e$ implies $y_{i}^{s}=0$ which contradicts the hypothesis that $y_{i}^{s}=z_{e}^{s}>0$. So $y_{i}^{s}=y_{i}^{s-1}$ implies $z_{e}^{s} \neq y_{i}^{s}$. This completes the proof of the lemma.

LEMmA 15. For all $e$ and $t$, if $z_{e}^{t}>0$ then

and

(a) $\boldsymbol{z}_{e}^{t} \in W_{e} \cap \overline{\alpha_{t}}$,

(b) $z_{e}^{t}=2 x$ implies $e_{x}>e$.

Proof. Assume $z=z_{e}^{t}>0$, and let $s^{\prime}$ be the least $s$ such that $z=z_{e}^{s}$. Then $z_{e}^{s^{\prime}}=z, s^{\prime} \leqq t$ and, by the construction, $s^{\prime} \equiv 2(\bmod 3)$, $z \in W_{e}^{s^{\prime}} \cap \overline{\alpha_{s^{\prime}}}$ and $z=2 x$ implies $e_{x}>e$. It remains to show $z \in \overline{\alpha_{t}}$. Suppose not, and let $t^{\prime}=$ the least $s$ such that $z \in \alpha_{t^{\prime}}, s^{\prime}<t^{\prime} \leqq t$. Then $t^{\prime} \equiv 1(\bmod 3)$ and $z \in E_{t^{\prime}} \cup O_{t^{\prime}}$. We claim that if $z \neq z_{e}^{t^{\prime}}$ then $z \neq z_{e}^{s}$ for all $s \geqq t^{\prime}$. Assume otherwise; i.e., $z \neq z_{e}^{t^{\prime}}$ but $z=z_{e}^{s}$ for some $s>t^{\prime}$. Let $s$ be least. Then $s>t^{\prime}, z_{e}^{s-1} \neq z=z_{e}^{s}>0$. Then $s \equiv 2(\bmod 3)$ and $z_{e}^{s}=z \in W_{e}^{s} \cap \overline{\alpha_{s}}$. But $s>t^{\prime}$ implies $z \in \alpha_{t^{\prime}} \subset \alpha_{s}$, which is a contradiction. So $z \neq z_{e}^{t^{\prime}}$ implies $z \neq z_{e}^{s}$ for all $s \geqq t^{\prime}$; so 
in particular $t \geqq t^{\prime}$ and $z \neq z_{e}^{t^{\prime}}$ implies $z \neq z_{e}^{t}$. But since by hypothesis $z=z_{e}^{t}$, it follows that $z=z_{e}^{t^{\prime}}$. Now by the construction, $t^{\prime} \equiv 1$ $(\bmod 3)$ and $z_{e}^{t^{\prime}-1} \neq z_{e}^{t^{\prime}}$ implies $z_{e}^{t^{\prime}}=0$. Since $z=z_{e}^{t^{\prime}}>0$, if follows that $z_{e}^{t^{\prime}-1}=z_{e}^{t^{\prime}}=z$. So $z_{e}^{t^{\prime \prime-1}}=z \notin E_{t^{\prime}}$, which implies $z \in O_{t^{\prime}}$. But then $z=z_{e}^{t^{\prime}-1}=y_{i}^{t^{\prime}-1}$ for some $i$, which contradicts Lemma 14 . So $t^{\prime}$ cannot exist, and $z \in \overline{\alpha_{t}}$.

DEFINITION 16. $z$ is permanently restrained by $e$ if $z>0$ and $z=\lim _{s} z_{e}^{s}$.

Lemma 17. For all $e$,

(a) If $z$ is permanently restrained by e, then $z \in \bar{\alpha}$.

(b) If $z$ is permanently restrained by e, then

$(\forall s)(\forall t)\left(z=z_{e}^{s}\right.$ and $\left.t>s \longrightarrow z=z_{e}^{t}\right)$.

(c) At most one $z$ is permanently restrained by $e$.

(d) If $z=z_{e}^{s}$ and $z \in \bar{\alpha}$, then $z$ is permanently restrained by $e$.

Proof. (a) Assume $z$ is permanently restrained by $e$. Then $z>0$ and $z=z_{e}^{s}$ for cofinitely many $s$. By Lemma 15(a), this implies $z \in \overline{\alpha_{s}}$ for cofinitely many $s$. So $z \in \bar{\alpha}$.

(b) Assume $z$ is permanently restrained by $e$ and $z=z_{e}^{s}$. Suppose $(\exists t)\left(t>s\right.$ and $\left.z \neq z_{e}^{t}\right)$ and let $t$ be least. Then $t>s$ and $z=z_{e}^{t-1} \neq z_{e}^{t}$. Then by Lemma 8 (a), since $z_{e}^{t-1}=z>0$ this implies $z \in \alpha$, which contradicts (a). So $(\forall t)\left(t>s \rightarrow z=z_{e}^{t}\right)$.

(c) Suppose $z$ is permanently restrained by $e$. Then $z=z_{e}^{s}$ for some $s$, and by (b), $z=z_{e}^{t}$ for all $t>s$. If $z^{\prime}$ is permanently restrained by $e$, then $z^{\prime}=z_{e}^{t}$ for cofinitely many $t$. Then in particular $z^{\prime}=z_{e}^{t}$ for some $t>s$, which implies $z^{\prime}=z$.

(d) Assume $z=z_{e}^{s}$ and $z \in \bar{\alpha}$. Since $0 \in \alpha$ it follows that $z>0$. Suppose that $(\exists t)\left(t>s\right.$ and $\left.z \neq z_{e}^{t}\right)$ and let $t$ be least. Then $0<z=$ $z_{e}^{t-1} \neq z_{e}^{t}$. Then by Lemma $8(\mathrm{a}), z=z_{e}^{t-1} \in \alpha$, contrary to hypothesis. So $(\forall t)\left(t>s \rightarrow z=z_{e}^{t}\right)$, i.e., $z=\lim _{s} z_{e}^{s}$ and $z$ is permanently restrained by $e$.

Lemma 18. For all $e$ and $z$,

(a) $\lim _{s} z_{e}^{s}$ exists.

(b) If $z(e)=\lim _{s} z_{e}^{s}, z(e)$ is recursive in $0^{\prime}$.

(c) $z$ is permanently restrained by $e$ if and only if $z=z(e)$ and $z(e)>0$.

Proof. Fix e. Since $D_{g(i)}$ is finite for each $i$, there exists $t$ such that $(\forall i)_{i \leq e}\left(D_{g(i)} \cap \alpha^{t}=D_{g(i)} \cap \alpha\right)$. Let $t(e)$ be the least such $t$; 
$t(e)$ is recursive in $0^{\prime}$ since $\alpha$ is r.e. and $\bigcup_{i \leqq e} D_{g(i)}$ is completely known from the canonical indexing. Define a function $r(e)$ by

$$
\begin{aligned}
r(e) & =1 & & \text { if } \quad(\exists s)\left(s \geqq t(e) \text { and } z_{e}^{s}>0\right) \\
& =0 & & \text { otherwise. }
\end{aligned}
$$

Then since $t(e)$ can be computed recursively in $0^{\prime}$ and $z_{e}^{s}$ is a recursive function of $s, r(e)$ is also recursive in $0^{\prime}$.

Case 1. $\quad r(e)=0$.

Then $(\forall s)\left(s \geqq t(e) \rightarrow z_{e}^{s}=0\right)$. Then $0=\lim _{s} z_{e}^{s}=z_{e}^{t(e)}$.

Case 2. $\quad r(e)=1$.

Then $(\exists s)\left(s \geqq t(e)\right.$ and $\left.z_{e}^{s}>0\right)$. Let $s(e)$ be the least such $s$. Then $s(e)$ is recursive in $0^{\prime}$, since $s(e)$ can in fact be computed recursively, given $t(e)$. Suppose $(\exists t)\left(t>s(e)\right.$ and $\left.z_{e}^{t} \neq z_{e}^{s(e)}\right)$, and let $t$ be least. Then $t>s(e), 0<z_{e}^{s(e)}=z_{e}^{t-1} \neq \boldsymbol{z}_{e}^{t}$. By Lemma 8(a), this implies $z_{e}^{s(e)}=z_{e}^{t-1} \in E_{t}$. Then by definition of $E_{t}$ it follows that $(\exists x)\left(z_{e}^{s(e)}=2 x\right.$ and $\left.(\forall i)_{i<e_{x}}\left(z_{e}^{s(e)} \neq z_{i}^{t-1}\right)\right)$. Since $z_{e}^{s(e)}=z_{e}^{t-1}$, this implies $e \geqq e_{x}$. But by Lemma $15(\mathrm{~b}), z_{e}^{s(e)}=2 x$ implies $e_{x}>e$, which gives a contradiction. So $z_{e}^{s(e)}=z_{e}^{t}$ for all $t \geqq s(e)$, and $z_{e}^{s(e)}=\lim _{s} z_{e}^{s}$.

Thus (a) holds in either case. Define

$$
\begin{aligned}
& z(e)=0 \quad \text { if } \quad r(e)=0 \\
& =s(e) \quad \text { if } \quad r(e)=1 .
\end{aligned}
$$

As shown above, $z(e)=\lim _{s} z_{e}^{s}$ for each $e$, and $z(e)$ is recursive in $0^{\prime}$ since $r(e)$ and $s(e)$ are, which proves (b). (c) is an immediate consequence of Definition 16 and the definition of $z(e)$.

Lemma 19. For all $e, e \in \gamma$ if and only if $\lim _{s} y_{e}^{s}=y$ exists and $y \in \bar{\alpha}$.

Proof. $(\hookleftarrow)$ Assume $y=\lim _{s} y_{e}^{s}$ and $y \in \bar{\alpha}$. Then $y>0$ since $0 \in \alpha$. Let $t$ be the least $s$ such that $y=y_{e}^{s}$. Then by the construction, $t \equiv 0(\bmod 3), e \leqq t$ and $x=h(y)$ implies $D_{\pi_{1}(x)} \subset \beta^{t}$ and $D_{\pi_{2}(x)} \subset \overline{\beta^{t}}$. Suppose $e \notin \gamma$. Then by Lemma $1, x \in W_{f(e)}$ and $D_{\pi_{1}(x)} \subset \beta$ implies $D_{\pi_{2}(x)} \cap \beta \neq \varnothing$, so there exists $s^{\prime}>t$ such that $D_{\pi_{2}(x)} \cap \beta^{s^{\prime}} \neq \varnothing$. Let $s^{\prime}$ be least, and choose $s^{\prime \prime} \geqq s^{\prime}$ such that $s^{\prime \prime} \equiv 1(\bmod 3)$. By Lemma $3(\mathrm{e}), 0<y=\lim _{s} y_{e}^{s}$ and $y=y_{e}^{t}$ implies $y=y_{e}^{s}$ for all $s \geqq t$; so in particular $s^{\prime \prime}>s^{\prime \prime}-1 \geqq s^{\prime}-1 \geqq t$ implies $y=y_{e}^{s^{\prime \prime}-1}=y_{e}^{s^{\prime \prime}}$. But $s^{\prime \prime} \geqq s^{\prime}>t$ also implies $e<s^{\prime \prime}$ and $D_{\pi_{2}(x)} \cap \beta^{s^{\prime \prime}} \neq \varnothing$. So by the construction $y=y_{e}^{s^{\prime \prime}-1} \in O_{s^{\prime \prime}}$ and $y_{e}^{s^{\prime \prime}}=O \neq y$, which is a contradiction. So $e \in \gamma$. 
$(\rightarrow)$ Assume $e \in \gamma$. Then by Lemma 1,

$(\exists x)\left(x \in W_{f(e)}\right.$ and $D_{\pi_{1}(x)} \subset \beta$ ane $\left.D_{\pi_{2}(x)} \subset \bar{\beta}\right)$. Let $x_{e}$ be the least such $x$, and let $s^{\prime}$ be the least $s$ such that $x_{e} \in W_{f(e)}^{s}$ and $D_{\pi_{1}}\left(x_{e}\right) \subset \beta^{s}$. Clearly $x_{e} \in W_{f(e)}^{s}$ and $D_{\pi_{1}\left(x_{e}\right)} \subset \beta^{\beta}$ and $D_{\pi_{2}\left(x_{e}\right)} \subset \overline{\beta^{s}}$ for all $s>s^{\prime}$. Since $x_{e}$ is least,

$$
(\forall x)_{x<x_{e}}\left(x \in W_{f(e)} \text { and } D_{\pi_{1}(x)} \subset \beta \longrightarrow D_{\pi_{2}(x)} \cap \beta \neq \varnothing\right) .
$$

Let $s^{\prime \prime} \geqq s^{\prime}$ be large that

$$
(\forall x)_{x<x_{e}}\left(x \in W_{f(e)} \text { and } D_{\pi_{1}(x)} \subset \beta \longrightarrow D_{\pi_{2}(x)} \cap \beta^{s^{\prime \prime}} \neq \varnothing\right) .
$$

Now by Lemma 18(a), $\lim _{s} z_{i}^{s}$ exists for all $i$. Let $m(i)$ be a function such that $z_{i}^{m(i)}=\lim _{s} z_{i}^{s}$, i.e.,

$$
(\forall i)(\forall s)\left(s \geqq m(i) \longrightarrow z_{i}^{s}=\boldsymbol{z}_{i}^{m(i)}\right),
$$

and choose $t \geqq s^{\prime \prime}, \max _{i<e} m(i)$.

Case 1. $(\forall s)\left(s \geqq t \rightarrow y_{e}^{s}>0\right)$.

Then $y_{e}^{t}>0$ so by Lemma 3(c), for all $t>s$ either $y_{e}^{s}=y_{e}^{t}$ or $y_{e}^{t^{\prime}}=0$ for some $t^{\prime}, s<t^{\prime} \leqq t$. Since by assumption the latter does not happen, it follows that $y_{e}^{t}=\lim _{s} y_{e}^{s}, y_{e}^{t}>0$.

Case 2. ( $\exists s)\left(s \geqq t\right.$ and $\left.y_{e}^{s}=0\right)$.

Let $t^{\prime}-1$ be the least such $s$; so $t^{\prime}>t, y_{e}^{t \prime-1}=0$. By the construction, if $t^{\prime} \not \equiv 0(\bmod 3)$ then $y_{e}^{t^{\prime}}=0$ also; so we may as well assume $t^{\prime} \equiv 0(\bmod 3)$. Then since $t^{\prime}>t \geqq s^{\prime \prime}, x_{e}$ is the least $x$ satisfying: $x \in W_{f(e)}^{t^{\prime}}$ and $D_{\pi_{1}(x)} \subset \beta^{t^{\prime}}$ and $D_{\pi_{2}(x)} \subset \overline{\beta^{t^{\prime}}}$. Since $y_{e}^{t^{\prime}-1}=0$ then by the construction $x_{e}^{t^{\prime}}=x_{e}, y_{e}^{t^{\prime}}>0$ and $x_{e}=h\left(y_{e}^{t^{\prime}}\right)$; let $y=y_{e}^{t^{\prime}}$. Assume $y \neq \lim _{s} y_{e}^{s}$; i.e., $y_{e}^{s} \neq y_{e}^{t^{\prime}}$ for some $s>t^{\prime}$. Let $s$ be least; then $s>t^{\prime}$, and $y=y_{e}^{t^{\prime}}=y_{e}^{s-1} \neq y_{e}^{s}$. Then by Lemma $3(b)$ either $y \in O_{s}$ or $y=z_{i}^{s}$ for some $i<e$. But $y \in O_{s}$ only if $D_{\pi_{2}(h(y))} \cap \beta^{s} \neq \varnothing$. Since $h(y)=x_{e}$ and $D_{\pi_{2}(x)} \subset \bar{\beta}$ this cannot happen. So $y \notin O_{s}$ which implies $y=z_{i}^{s}$ for some $i<e$. But by Lemma 14, $0<y=y_{e}^{s-1}$ implies $y \neq z_{i}^{s-1}$. So $i<e$ and $z_{i}^{s-1} \neq z_{i}^{s}$. But $s>t^{\prime}>t>\max _{i<e} m(i)$, so $s-1 \geqq m(i)$ which implies $z_{i}^{s}=z_{i}^{s-1}=z_{i}^{m(i)}$ which is a contradiction. It follows that $y=\lim _{s} y_{e}^{s}$, where $y>0$.

Thus in either case $e \in \gamma$ implies $\lim _{s} y_{e}^{s}$ exists and $\lim _{s} y_{e}^{s}=y>0$. To show $y \in \bar{\alpha}$, it suffices to observe that by Lemma 4 (a), $y_{e}^{s}>0$ implies $y_{e}^{s} \in \overline{\alpha_{s}}$; so $y \in \overline{\alpha_{s}}$ for cofinitely many $s$, which can happen only if $y \in \bar{\alpha}$. 
LEMMA 20. Let

$$
\begin{aligned}
y(e) & =0 \quad \text { if } e \notin \gamma, \\
& =\lim _{s} y_{e}^{s} \quad \text { if } e \in \gamma .
\end{aligned}
$$

Then $y(e)$ is defined for all e, and $y(e)$ is recursive in $\gamma$.

Proof. It follows from Lemma 19 that $y(e)$ is defined for all $e$. To compute $y(e)$ recursively in $\gamma$, ask first whether $e \in \gamma$. If not, $y(e)=0$. If $e \in \gamma$, it remains to compute $\lim _{s} y_{e}^{s}$. But this can be done recursively in $0^{\prime}$, since $y_{e}^{s}$ is a recursive function of $s$, and

$$
\begin{aligned}
y=\lim _{s} y_{e}^{s} \longleftrightarrow(\exists s)(\forall t)\left(t \geqq s \longrightarrow y=y_{e}^{t}\right) \\
\longleftrightarrow(\forall s)(\exists t)\left(t \geqq s \text { and } y=y_{e}^{t}\right) .
\end{aligned}
$$

Since by hypothesis $\gamma \in \boldsymbol{c}$ and $\mathbf{0}^{\prime} \leqq \boldsymbol{c}, y(e)$ can be computed recursively in $\gamma$.

LEMma 21. $W_{e} \cap \bar{\alpha} \neq \varnothing$ if and only if there is a $z$ in $W_{e}$ satisfying one of the following conditions:

(a) $(\exists i)_{i \leqq e}(z$ is permanently restrained by $i)$,

(b) $(\exists i)_{i \leqq e}\left(i \in \gamma\right.$ and $\left.z=\lim _{s} y_{i}^{s}\right)$,

(c) $(\exists i)_{i \leqq e}\left(i \in \bar{\beta}\right.$ and $\left.z \in D_{g(i)}\right)$.

Proof. (৬) Assume that for some $z$ in $W_{e}$, (a), (b) or (c) holds. If $z$ is permanently restrained by $i$, then $z \in \bar{\alpha}$ by Lemma 17(a). If $z=\lim _{s} y_{i}^{s}$ for some $i \in \gamma$, then $z \in \bar{\alpha}$ by Lemma 19. If $z \in D_{g(i)}$ for $i \in \bar{\beta}, z=2 x$ for some $x>0$ and $i=e_{x} \in \bar{\beta}$ by Definition 2. Now $0<z \in \alpha$ only if $z \in E_{s} \cup O_{s}$ for some $s$. But $z \in E_{s}$ implies $e_{x} \in \beta$, and $z \in O_{s}$ implies $z$ is odd. Since neither is the case, $z \notin E_{s} \cup O_{s}$ and $z \in \bar{\alpha}$. Thus in all cases, $z \in \bar{\alpha} \cap W_{e}$ and $W_{e} \cap \bar{\alpha} \neq \varnothing$.

$(\rightarrow)$ Assume $W_{e} \cap \bar{\alpha} \neq \varnothing$. Let $z$ be the least element of $W_{e} \cap \bar{\alpha}$, and let $s^{\prime}$ be the least $s$ such that $z \in W_{e}^{s}$, then $z \in W_{e}^{s} \cap \bar{\alpha}_{s}$ for all $s \geqq s^{\prime}$. Since $0 \in \alpha, z>0$ and since $z$ is least, it follows that $(\forall y)_{y<z}\left(y \in W_{e} \rightarrow y \in \alpha\right)$. Thus for each $y<z$ such that $y \in W_{e}$ there is a stage $s(y)$ such that $y \in \alpha^{s(y)}$. Choose $t \geqq s^{\prime}, \max _{y<z} s(y)$.

Case 1. $(\forall s)\left(s \geqq t \rightarrow z_{e}^{s}>0\right)$.

Let $y=z_{e}^{t}$. Then $y>0$ and by Lemma 15(a), $y \in W_{e}$. Suppose that $z_{e}^{s} \neq y$ for some $s>t$, and let $s$ be least. Then

$$
0<y=z_{e}^{s-1} \neq z_{e}^{s}
$$

which by Lemma 8 (a) implies $z_{e}^{s}=0$, which contradicts the assump- 
tion since $s>t$. It follows that $y=\lim _{s} z_{e}^{s}$ so that $y \in W_{e}$ is permanently restrained by $e$. So (a) holds.

Case 2. $(\exists s)\left(s \geqq t\right.$ and $\left.z_{e}^{s}=0\right)$.

Let $t^{\prime}-1$ be any such $s$; so $t^{\prime}>t$ and $z_{e}^{t^{\prime-1}}=0$. If $t^{\prime} \not \equiv 2(\bmod 3)$ then by the construction $z_{e}^{t^{\prime}}=0$ also, so we may as well assume $t^{\prime} \equiv 2(\bmod 3)$. Since $t^{\prime}-1 \geqq t \geqq s^{\prime}$ we have $z \in W_{e}^{t^{\prime}} \cap \overline{\alpha_{t^{\prime}}}$. We consider several subcases.

Subcase 2.1. $(\forall i)_{i \leqq e}\left(z \neq y_{i}^{t^{\prime}-1}\right)$ and $(\forall x)_{x<z}\left(z=2 x \rightarrow e_{x}>e\right)$.

Now since $t^{\prime}>t>\max _{y<z} s(y)$ it follows that no $y<z$ is in $W_{e}^{t^{\prime}} \cap \overline{\alpha_{t^{\prime}}}$. So $z$ is the least $z$ satisfying the requirements of the construction for choosing a new value of $z_{e}^{t^{\prime}}$ when $t^{\prime} \equiv 2(\bmod 3)$. So $z=z_{e}^{t^{\prime}}$. Since by hypothesis $z \in \bar{\alpha}$, this implies by Lemma 17(d) that $z$ is permanently restrained by $e$. So (a) holds.

Subcase 2.2. $(\exists i)_{i \leqq e}\left(z=y_{i}^{t^{\prime}-1}\right)$ or $(\exists x)_{x<z}\left(z=2 x\right.$ and $\left.e_{x} \leqq e\right)$.

Subcase 2.2.1. $\quad(\exists i)_{i \unlhd e}\left(z=y_{i}^{t^{\prime}-1}\right)$.

Let $i$ be least, $i \leqq e$. If $z=\lim _{s} y_{i}^{s}$ then by Lemma 19, $z \in \bar{\alpha}$ implies $i \in \gamma$, in which case (b) holds. If $z \neq \lim _{s} y_{i}^{s}$, then $(\exists s)\left(s \geqq t^{\prime}\right.$ and $\left.z \neq y_{i}^{s}\right)$; let $s$ be least. Then $0<z=y_{i}^{s-1} \neq y_{i}^{s}$ which by Lemma 3(b) implies $z \in O_{s} \subset \alpha$ or $z=z_{j}^{s}$ for some $j<i$. The former cannot happen, since $z \in \bar{\alpha}$; so $z=z_{j}^{s}$ for $j<i \leqq e$. Then by Lemma $17(\mathrm{~d})$, $z$ is permanently restrained by $j<e$, and (a) holds.

Subcase 2.2.2. $(\forall i)_{i \leqq e}\left(z \neq y_{i}^{t^{\prime}-1}\right)$ and $(\exists x)_{x<z}\left(z=2 x\right.$ and $\left.e_{x} \leqq e\right)$.

Let $x=z / 2$; then $e_{x} \leqq e$ and $z \in D_{g\left(e_{x}\right)}$ by Definition 2. If $e_{x} \in \bar{\beta}$, then (c) holds with $i=e_{x}$. Now assume $e_{x} \in \beta$. Then $e_{x} \in \beta^{s}$ for some $s$; let $s^{\prime \prime}$ be the least such $s$. Choose $t^{\prime \prime}$ so large that

$$
t^{\prime \prime}>\max \left\{s^{\prime \prime}, t^{\prime}, e_{x}\right\}
$$

and $t^{\prime \prime} \equiv 1(\bmod 3)$. Suppose that $(\forall i)_{i<e_{x}}\left(z \neq z_{i}^{t^{\prime \prime}-1}\right)$. Then since $e_{x} \leqq t^{\prime \prime}$ and $e_{x} \in \beta^{s^{\prime \prime}} \subset \beta^{t^{\prime \prime}}$, if follows that $z \in E_{t^{\prime \prime}} \subset \alpha$, which contradicts the hypothesis that $z \in \bar{\alpha}$. So $z=z_{i}^{t^{\prime \prime}-1}$ for some $i<e_{x}$. But then it again follows by Lemma $17(\mathrm{~d})$ that $z$ is permanently restrained by $i<e_{x} \leqq e$, and (a) holds.

This completes the proof of the lemma.

LEMMA 22. $\theta A^{\alpha} \leqq{ }_{T} \gamma$.

Proof. We show how to decide for each $e$ whether $W_{e} \subset \alpha$, recursively in $\gamma$. By Lemma 18, there is a function $z(i)$ recursive in 
$0^{\prime}$ such that $z$ is permanently restrained by $i$ if and only if

$$
z=z(i)>0 \text {. }
$$

By Lemma 20, there is a function $y(i)$ recursive in $\gamma$ such that for each $i, y(i)=\lim _{s} y_{i}^{s}$ if $i \in \gamma$. Let

$$
\begin{aligned}
& T_{e}=\{z(i) \mid i \leqq e \text { and } z(i)>0\}, \\
& U_{e}=\{y(i) \mid i<e \text { and } i \in \gamma\}, \\
& V_{e}=\left\{z \mid(\exists i)_{i<e}\left(i \in \bar{\beta} \text { and } z \in D_{g(i)}\right\} .\right.
\end{aligned}
$$

The sets $T_{e}, U_{e}, V_{e}$ are evidently finite. The membership of $T_{e}$ can be completely determined recursively in $0^{\prime}$ and hence in $\gamma$ since $\gamma \in \boldsymbol{c}$ and $0^{\prime} \leqq c$. The membership of $U_{e}$ can be determined recursively in $\gamma$. The membership of $V_{e}$ can be obtained recursively in $\beta$ and hence recursively in $\gamma$, since $\beta \in \boldsymbol{b} \leqq 0^{\prime} \leqq c$. Since this can all be done uniformly given $e$, there is a function $q(e)$, recursive in $\gamma$, such that $T_{e} \cup U_{e} \cup V_{e}=D_{q(e)}$, for each $e$. Now by Lemma 21,

$$
\begin{aligned}
W_{e} \cap \bar{\alpha} \neq \varnothing & \longleftrightarrow(\exists z)\left(z \in W_{e} \text { and } z \in T_{e} \cup U_{e} \cup V_{e}\right) \\
& \longleftrightarrow V_{e} \cap D_{q(e)} \neq \varnothing .
\end{aligned}
$$

But once $q(e)$ is known, the question of whether $W_{e} \cap D_{q(e)}=\varnothing$ can be answered recursively in $\mathbf{0}^{\prime}$, and hence recursively in $\gamma$.

Lemma 23. For each e, let

$$
\begin{aligned}
& T_{e}=\{z \mid z \text { is permanently restrained by some } i<e\}, \\
& R_{e}=\left\{y \mid y>0 \text { and }(\exists s)\left(y=y_{e}^{s}\right)\right\} .
\end{aligned}
$$

Then $e \in \gamma$ if and only if $R_{e} \cap \bar{\alpha} \cap \bar{T}_{e} \neq \varnothing$.

Proof. $(\rightarrow)$. Asume $e \in \gamma$. Then by Lemma 19, $\lim _{s} y_{e}^{s}=y$ exists and $y \in \bar{\alpha}$. Clearly $y \in R_{e}$, so it suffices to show $y \notin T_{e}$. Suppose $y$ is permanently restrained by some $i<e$. Then $y=z_{i}^{s}$ for cofinitely many $s$. But $y=\lim _{s} y_{e}^{s}$ implies $y=y_{e}^{s}$ for cofinitely many $s$; while by Lemma $14,0<y=z_{i}^{s}$ implies $y \neq y_{e}^{s}$ for all $s$, which is a contradiction. So $y \in R_{e} \cap \bar{\alpha} \cap \bar{T}_{e}$.

$(\leftarrow)$ Assume $R_{e} \cap \bar{\alpha} \cap \bar{T}_{e} \neq \varnothing$, and let $y$ be the least element of $R_{e} \cap \bar{\alpha} \cap \bar{T}_{e}$. Now $y \in R_{e}$ implies $0<y=y_{e}^{t}$ for some $t$. Since $y \in \bar{\alpha}$ it suffices by Lemma 19 to show $y=\lim _{s} y_{e}^{s}$. Suppose not; then for some $s>t, y_{e}^{s} \neq y_{e}^{t}=y$; and if $s$ is least, $0<y=y_{e}^{s-1} \neq y_{e}^{s}$. Then by Lemma $3(\mathrm{~b})$, either $y \in O_{s} \subset \alpha_{s}$ or $y=z_{i}^{s}$ for some $i<e$. But $y \in \alpha_{s}$ contradicts the hypothesis that $y \in \bar{\alpha}$; while $0<y=z_{i}^{s}$ for $i<e$ 
implies by Lemma 17(d) that $y$ is permanently restrained by $i<e$, contradicting the hypothesis that $y \notin T_{e}$. So $y=\lim _{s} y_{e}^{s}$ and $y \in \bar{\alpha}$ which implies $e \in \gamma$.

Lemma 24. $\gamma \leqq{ }_{T} \theta A^{\alpha}$.

Proof. Let

$$
\begin{aligned}
& T_{e}=\{z \text { is permanently restrained by some } i<e\}, \\
& R_{e}=\left\{y \mid y>0 \text { and }(\exists s)\left(y=y_{e}^{s}\right)\right\} .
\end{aligned}
$$

By Lemma 23, $e \in \gamma$ if and only if $R_{e} \cap \bar{\alpha} \cap \bar{T}_{e} \neq \varnothing$. So it suffices to show that we can decide whether $R_{e} \cap \bar{\alpha} \cap \bar{T}_{e}=\varnothing$, recursively in $\theta A^{\alpha}$. By Lemma 13, $\beta \leqq{ }_{T} \alpha$. This implies $\alpha$ is nonrecursive since $\beta$ is nonrecursive. In particular, $\alpha \neq \varnothing$ or $N$, so $A^{\alpha}$ is nontrivial and by Rice's theorem, [4], $0^{\prime}$ is recursive in $\theta A^{\alpha}$. Since $y_{e}^{s}$ is a recursive function of $s, R_{e}$ is an r.e. set. By Lemma 18,

$$
T_{e}=\{z(i) \mid i<e \quad \text { and } z(i)>0\}
$$

where $z(i)$ is recursive in $0^{\prime}$ and thus in $\theta A^{\alpha}$. Thus the membership of the finite set $T_{e}$ can be completely determined, recursively in $\theta A^{\alpha}$, and since this can be done uniformly in $e$, there is a function $p(e)$ recursive in $\theta A^{\alpha}$ such that $W_{p(e)}=R_{e}-T_{e}$, for cach $e$. Then

$$
\begin{aligned}
e \in \gamma & \longleftrightarrow W_{p(e)} \cap \bar{\alpha} \neq \varnothing \\
& \longleftrightarrow p(e) \notin \theta A^{\alpha},
\end{aligned}
$$

which can be decided recursively in $\theta A^{\alpha}$ once $p(e)$ is known. This completes the proof of the lemma.

It follows from Lemmas 11 and 13 that $\alpha \in \boldsymbol{b}$, and by Lemmas 22 and 24 that $\theta A^{\alpha} \in c$. Since $\alpha$ is r.e., this completes the proof of the theorem.

\section{REFERENCES}

1. J. C. E. Dekker and J. Myhill, Some theorems on classes of recursively enumerable sets, Trans. Amer. Math. Soc., 89 (1958), 25-59.

2. L. Hay, Discrete $\omega$-sequences of index sets, to appear in Trans. Amer. Math. Soc.

3. T. G. McLaughlin, The family of all recursively enumerable classes of finite sets, Trans. Amer. Math. Soc., 155 (1971), 127-136.

4. D. A. Martin, Completeness, the recursion theorem, and effectively simple sets, Proc. Amer. Math. Soc., 17 (1966), 838-842.

5. H. Rogers, Jr., Theory of Recursive Functions and Effective Computability, McGraw Hill, New York, 1967.

6. G. E. Sacks, Degrees of Unsolvability, Ann. of Math. Studies No. 55, Princeton University Press, Princeton, New Jersey, 1963. 
7. C. E. M. Yates, Three theorems on the degrees of recursively enumerable sets, Duke Math. J., 32 (1965), 461-468.

Received January 2, 1972. Research supported in part by National Science Foundation Grant GP-19958.

The University of Illinois at Chicago Circle 



\section{PACIFIC JOURNAL OF MATHEMATICS}

\section{EDITORS}

D. Gilbarg and J. Milgram Stanford University

Stanford, California 94305

R. A. Beaumont

University of Washington

Seattle, Washington 98105
J. DugundJI

Department of Mathematics

University of Southern California

Los Angeles, California 90007

RICHARD ARENS

University of California

Los Angeles, California 90024

ASSOCIATE EDITORS
E. F. BECKENBACH
B. H. NeumanN
F. WOLF
K. YoSHIDA

\section{SUPPORTING INSTITUTIONS}

UNIVERSITY OF BRITISH COLUMBIA

CALIFORNIA INSTITUTE OF TECHNOLOGY

UNIVERSITY OF CALIFORNIA

MONTANA STATE UNIVERSITY

UNIVERSITY OF NEVADA

NEW MEXICO STATE UNIVERSITY

OREGON STATE UNIVERSITY

UNIVERSITY OF OREGON

OSAKA UNIVERSITY
UNIVERSITY OF SOUTHERN CALIFORNIA

STANFORD UNIVERSITY

UNIVERSITY OF TOKYO

UNIVERSITY OF UTAH

WASHINGTON STATE UNIVERSITY

UNIVERSITY OF WASHINGTON

$\stackrel{*}{*} \stackrel{*}{*} \stackrel{*}{*}$ AMERICAN MATHEMATICAL SOCIETY

NAVAL WEAPONS CENTER 


\section{Pacific Journal of Mathematics}

\section{Vol. 46, No. $1 \quad$ November, 1973}

Allan Francis Abrahamse, Uniform integrability of derivatives on $\sigma$-lattices .......................................... 1

Ronald Alter and K. K. Kubota, The diophantine equation $x^{2}+D=p^{n} \ldots \ldots \quad 11$

Grahame Bennett, Some inclusion theorems for sequence spaces .......... 17

William Cutler, On extending isotopies ........................ 31

Robert Jay Daverman, Factored codimension one cells in Euclidean

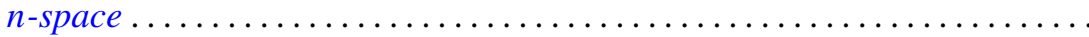

Patrick Barry Eberlein and Barrett O’Neill, Visibility manifolds ............ 45

M. Edelstein, Concerning dentability .......................... 111

Edward Graham Evans, Jr., Krull-Schmidt and cancellation over local

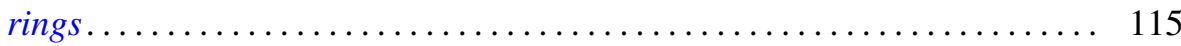

C. D. Feustel, A generalization of Kneser's conjecture ................ 123

Avner Friedman, Uniqueness for the Cauchy problem for degenerate parabolic equations .......................................... 131

David Golber, The cohomological description of a torus action ............ 149

Alain Goullet de Rugy, Un théorème du genre "Andô-Edwards" pour les

Fréchet ordonnés normaux..............................

Louise Hay, The class of recursively enumerable subsets of a recursively enumerable set ........................................

John Paul Helm, Albert Ronald da Silva Meyer and Paul Ruel Young, On orders of translations and enumerations...

Julien O. Hennefeld, A decomposition for $B(X)^{*}$ and unique Hahn-Banach

extensions

Gordon G. Johnson, Moment sequences in Hilbert space .

Thomas Rollin Kramer, A note on countably subparacompact spaces ...

Yves A. Lequain, Differential simplicity and extensions of a derivation ....

Peter Lorimer, A property of the groups Aut $\mathrm{PU}\left(3, q^{2}\right) \ldots$

225

Yasou Matsugu, The Levi problem for a product manifold.

231

John M.F. O'Connell, Real parts of uniform algebras ......

William Lindall Paschke, A factorable Banach algebra without bounded

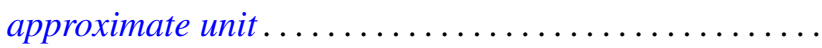

Ronald Joel Rudman, On the fundamental unit of a purely cubic field ....

Tsuan Wu Ting, Torsional rigidities in the elastic-plastic torsion of simply connected cylindrical bars .........................

Philip C. Tonne, Matrix representations for linear transformations on analytic sequences...................................

Jung-Hsien Tsai, On E-compact spaces and generalizations of perfect

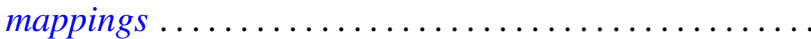

Alfons Van Daele, The upper envelope of invariant functionals majorized by an invariant weight. .. 